\title{
SPATIAL-TEMPORAL ANALYSIS OF SOCIAL MEDIA DATA RELATED TO NEPAL EARTHQUAKE 2015
}

\author{
L. Thapa* \\ Survey Officer, Survey Department, Kathmandu, Nepal - thapalaxmi278@gmail.com
}

KEY WORDS: Spatial, Temporal, Geoweb, Location Based Social Media, Twitter, Nepal Earthquake 2015

\begin{abstract}
:
Social Medias these days have become the instant communication platform to share anything; from personal feelings to the matter of public concern, these are the easiest and aphoristic way to deliver information among the mass. With the development of Web 2.0 technologies, more and more emphasis has been given to user input in the web; the concept of Geoweb is being visualized and in the recent years, social media like Twitter, Flicker are among the popular Location Based Social Medias with locational functionality enabled in them. Nepal faced devastating earthquake on 25 April, 2015 resulting in the loss of thousands of lives, destruction in the historical-archaeological sites and properties. Instant help was offered by many countries around the globe and even lots of NGOs, INGOs and people started the rescue operations immediately; concerned authorities and people used different communication medium like Frequency Modulation Stations, Television, and Social Medias over the World Wide Web to gather information associated with the Quake and to ease the rescue activities. They also initiated campaign in the Social Media to raise the funds and support the victims. Even the social medias like Facebook, Twitter, themselves announced the helping campaign to rebuild Nepal. In such scenario, this paper features the analysis of Twitter data containing hashtag related to Nepal Earthquake 2015 together with their temporal characteristics, when were the message generated, where were these from and how these spread spatially over the internet?
\end{abstract}

\section{INTRODUCTION}

Technological advancement in the field of mobile devices, especially smart phones, availability of ubiquitous internet connectivity and positioning GPS sensors have revolutionize how people access to global information. Global social media research summary 2016 shows, out of 7.395 billion population of the world as of January 2016, 3.419 billion people are using internet and 2.307 billion people are active social media users (Chaffey 2016). Facebook received 1.04 billion daily active users on average for December 2015 (Facebook 2015). Statistics shows that Facebook is the most used social networking sites with $87.69 \%$ usage followed by Twitter with $6.43 \%$ of usage. Pininterest stands third with $4.55 \%$ and Tumblr stands at fourth position with $0.43 \%$ of usage (StatsMonkey 2015). In Nepal, social media like Facebook, Twitter, Flickr, and many more has replaced postal system as the swift communication medium. Record shows that 5,700,000 people have access to internet and are using Facebook as of November 15, 2015 (Internet World Stats 2015) and more than one million people are using Twitter in Nepal.

This growing statistics of people accessing internet and social media has opened wider applications of the same. Besides connecting people with each other, social media are making them abreast with the world just in few clicks in a matter of seconds. These are no more confined as the platform to share temperament or promote business; but are nowadays becoming the effective information podium for emergency rescue and recovery during the disaster. Twitter, with its word limit of 140, is one of the quick and effective way to share such information.

When Earthquake of magnitude 7.8 Mw hit Nepal on the day of 25th April 2015, overstretched telephone communications with heavy network traffic made people difficult to connect with each other. Fortunately, communications infrastructures was not damaged and access to the internet communication allowed citizens to communicate with their families and lived ones using Facebook, Twitter and other social media (Thapa, 2016). Notably, many rescue operations were carried out on the basis of information posted on those social networking sites. The most popular social media of the country, Facebook deployed "Safe Check" feature in it; more than 7 million people were marked safe and more than 150 million friends received notifications informing their friend were safe during the activation period (Zuckerberg 2015, Wikipedia 2016). It was a big start to support disaster responders focus on rescue operations. In twitter, standard hashtags like \#NepalEarthquake, \#NepalQuakeRelief, \#rapidresponseteam, and many more were being tweeted along with pictures, videos and other information to spread message about earthquake to the concerned authority and ease the rescue operations. Even many organizations and people used these sites for fund collection and recovery activities. This paper discusses the spatial temporal dissemination of twitter during the period of earthquake; where and when the message were generated?

\section{METHODOLOGY}

\subsection{Data Collection}

Twitter data collected by The Center of Human Dynamics in the Mobile Age (HDMA) at San Diego State University had been used for the analysis. After the Mega Earthquake 2015 hit Nepal

"Laxmi Thapa 
badly, the HDMA Center created user friendly and easy to use GeoViewer for Nepal Earthquake

(http://humandynamics.sdsu.edu/NepalEarthquake.html) on April 26, 2015 allowing real-time monitoring of tweets and analysis of tweets collected during and after the disaster (HDMA, 2016).

Information about the occurrence of Earthquake at various time period was collected from the website of National Seismological Centre, Nepal ${ }^{1}$. The collected data contained locational information of epicenter of the earthquake occurred from 25 April to 18 May, 2015 in terms of latitude and longitude, magnitude of earthquake and date and time of the earthquake occurrence.

\subsection{Data Analysis}

Only the tweets with hashtags, containing information related to earthquake were considered for the analysis to reduce the irrelevant information. Following methods were used to analyze the collected tweets.

\section{Distance from Epicentre}

The distance from epicenter to the tweets generated was calculated by using Point Distance Function in ArcGIS 10.3. Also, closest distance was calculated in ArcGIS 10.3 by using:

Generate Near Table: It calculates nearest distance and generates a new table with the information of Field Id and Nearest distance

Near: It calculates nearest distance and updates the input layer with near Field Id, near distance and near X and Y co-ordinate.

Time at which Earthquake occurred and the tweets generated

Tweets retrieved contained information about the time when users tweeted it and were compared with the time when the earthquake occurred.

\section{Follower, Friends and Status on Twitter}

The way information is disseminated effectively can be seen by the no. of times the tweets is being retweeted, the no. of follower and friends the user has on his/her twitter account.

The data has no information about like tweets (favorite) and retweets which are important indication to see the effective communication/information dissemination.

\section{FINDINGS AND DISCUSSION}

\subsection{Findings}

The tweets used for this study includes tweets generated from 20:04pm, April 252015 to 23: 59pm, May 18 2015. There were altogether 33,610 tweets containing temporal information showing local time at which the tweet was generated, text, urls, number of followers and friends, status count, geographical location, source from which the information generated, and language used. Out of those, only 7,713 tweets were tweeted mentioning hashtags and the remaining 25,897 tweets were without hashtag. Among the 7,713 tweets with the hashtags, only 5,618 tweets contained information related to earthquake and were considered for the further analysis.

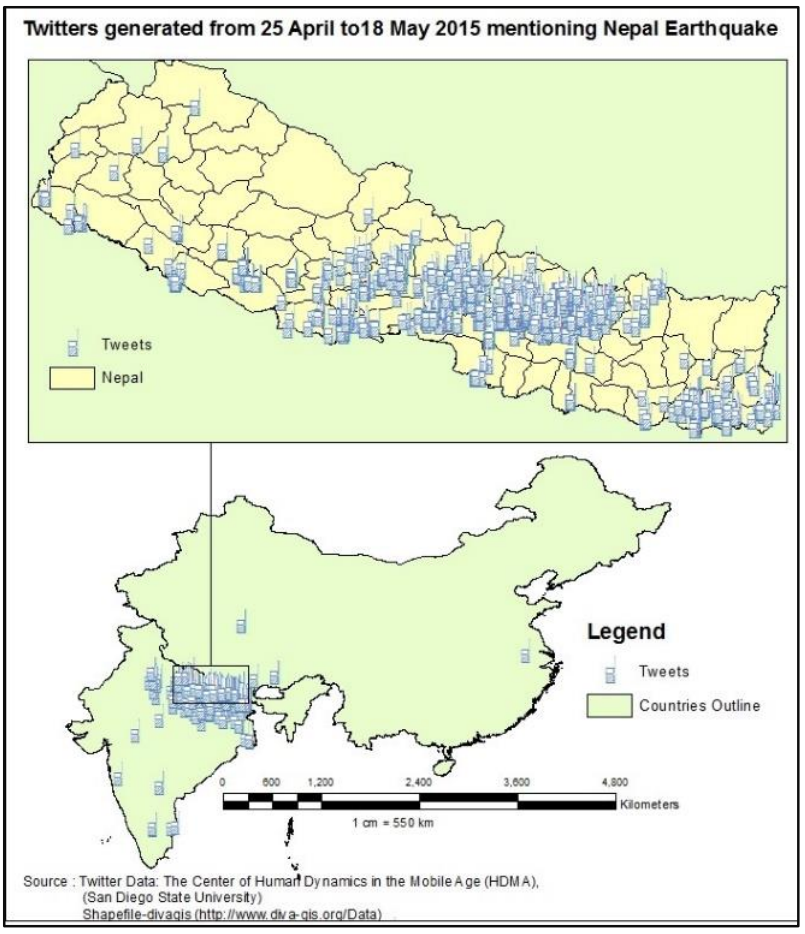

Figure 1: twitter generated from different countries from 25 April to 18 May, 2015.

The data shows that people mostly preferred English language for the tweets followed by native Nepali language. Of the 5,618 tweets analyzed, 1699 tweets contained URLS along with the other information.

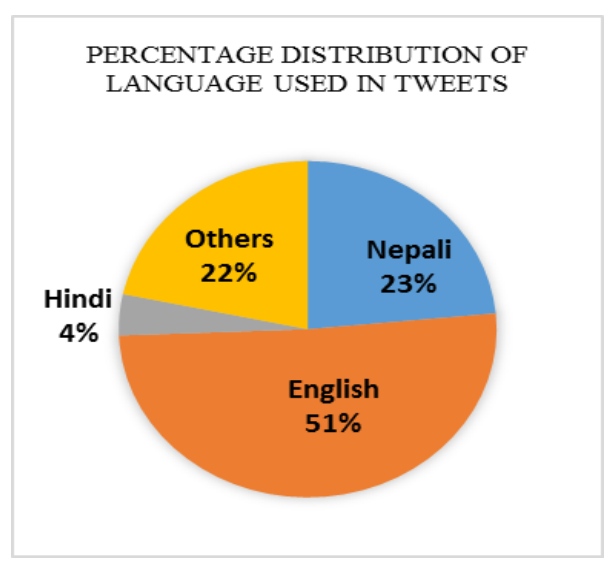

Figure 2: Percentage Distribution of language used in the tweets.

During the tremor, few hashtags used were standard and initiated by organizations working in the rescue and recovery activities and others were used by individuals in their own way. Many hashtags were representing same information but were tweeted in different way; representative examples include \#earthquakenepal and \#NepalEarthquake, \#NepalEarthquakeRelief and \#NepalQuakeRelief, \#Pray4Nepal and \#prayfornepal. Table below shows tweets the 14 most popular hashtags and number of times the hashtags were tweeted: 


\begin{tabular}{|l|l|l|}
\hline S.No. & Hashtag & $\begin{array}{l}\text { No. of } \\
\text { Occurrence }\end{array}$ \\
\hline 1 & Nepal & 2203 \\
\hline 2 & Earthquake & 1023 \\
\hline 3 & NepalEarthquake & 875 \\
\hline 4 & NepalQuake & 744 \\
\hline 5 & GoHomeIndianMedia & 518 \\
\hline 6 & HelpNepal & 296 \\
\hline 7 & Prayfornepal & 283 \\
\hline 8 & NepalQuakeVictims & 122 \\
\hline 9 & earthquakenepal & 91 \\
\hline 10 & EarthquakeAgain & 68 \\
\hline 11 & rapidresponseteam & 62 \\
\hline 12 & Gorkha & 58 \\
\hline 13 & EarthQuakeResponse & 56 \\
\hline 14 & NepalQuakeRelief & 55 \\
\hline
\end{tabular}

Table 3: Hashtags tweeted greater than 50 times.

\section{Distance from Epicentre}

The epicenter of First earthquake occurred on April 25, 2015 at 11: 56 was at Gorkha with the geographical co-ordinates of 28.24, 84.75. The major earthquake of April 25 was followed by 243 post quakes till 18 May. April 25 and 26 encountered 38 quakes followed by 36 on May 12, which faced earthquake of $6.8 \mathrm{Mw}$, biggest after April 25 (7.6 Mw) and April 26 (6.9 Mw).

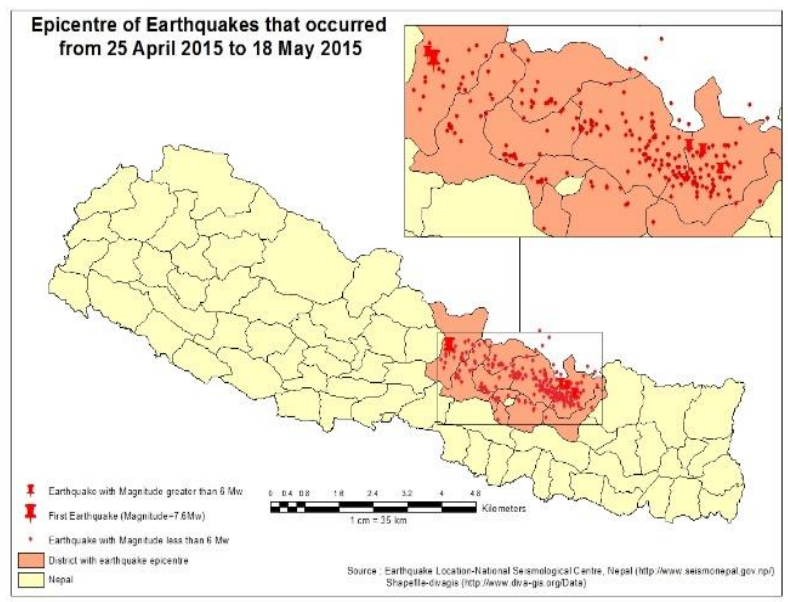

Figure 4: Earthquake occurrence from 25 April to 18 May, 2015

The nearest tweets occurred twice at the distance of 982.67 meter at 9:59 am and 8:58 am on 27 April. The farthest tweet was at the distance of 3,730,263.9 meter tweeted at 19:21 pm on 9 May 2015.

Time at which Earthquake occurred and the tweets generated

The time at which first Earthquake occurred at Gorkha was 11:56 am. The first tweet retrieved for the analysis was at 20:02 followed by 237 tweets the same day; 26 April, the day after the Earthquake, that itself encountered postquakes of $6.9 \mathrm{Mw}$ had the highest number of tweets counting 714 disseminating information about earthquake using different hashtags and was followed by 590 tweets on 3 May, whereas 11 May that faced the postquake of $6.8 \mathrm{Mw}$, had 511 tweets.

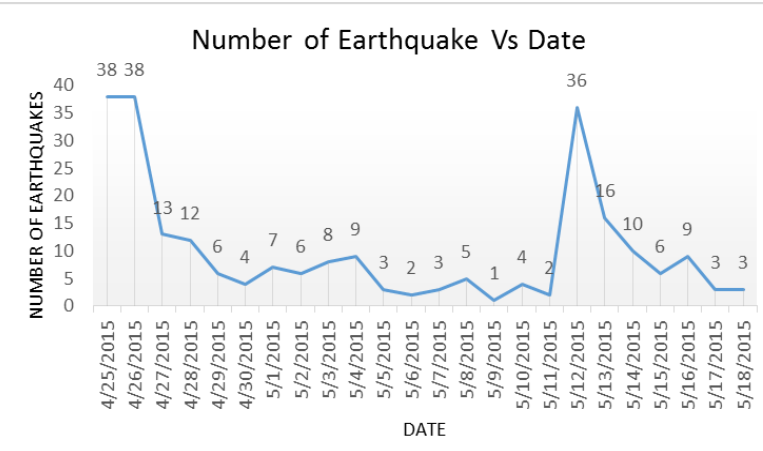

Figure 5: Number of earthquake occurred from 25 April to 18 May, 2015.

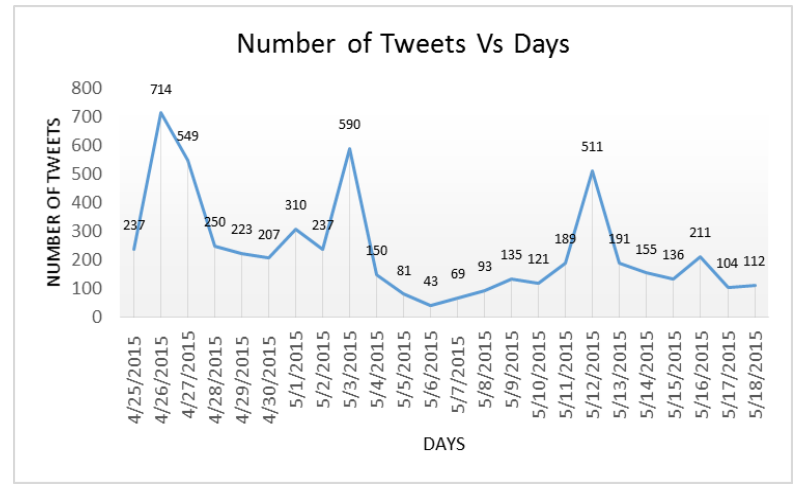

Figure 6: Number of tweets tweeted from 25 April to 18 May, 2015.

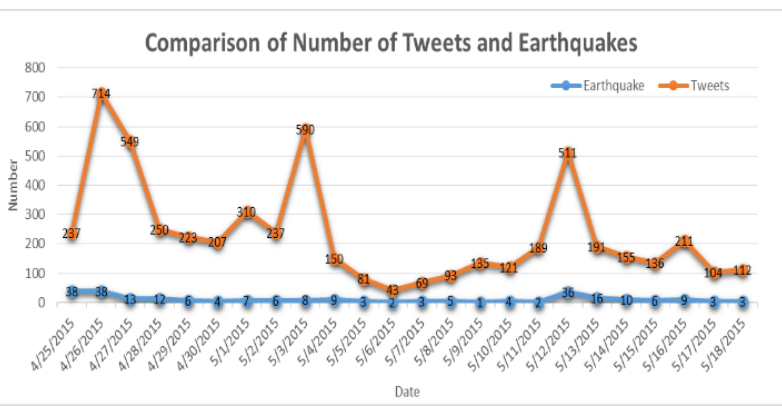

Figure 7: Comparison of Tweets and earthquake occurred from 25 April to 18 May, 2015 


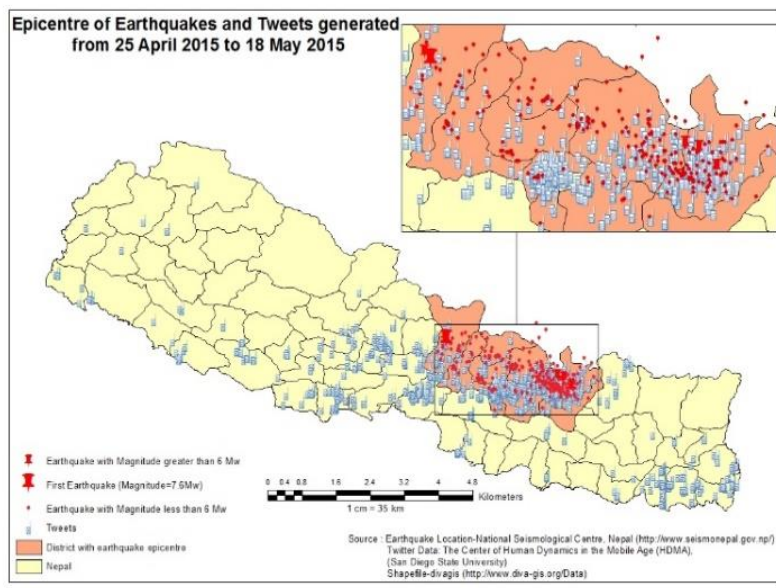

Figure 8: Earthquake and Twitter from 25 April to 18 May, 2015.

\section{Friends, Followers and Status}

Data retrieved shows one of the user had 128623 Tweets follower making it the highest number of followers and 16744 Status tweeted yet; user with 127919 follower and 16658 tweet status stood second, followed by 120784 followers and 2787 status at the third place. Whereas, there were users with no followers, no friends, but single tweet status. Since the data retrieved contained no retweeted information to represent effective information dissemination, no further analysis was done.

\subsection{Discussion}

The results show that not all the people using twitter used Hashtag; only 22 percentage of the people used different Hashtags and not all the tweets with the hashtags contain information about the earthquake. Most of the tweets containing Hashtag with the information about the earthquake are from the districts affected with the earthquake. Also, people from Eastern terai and mid-hills were using twitter mentioning earthquake in their tweets and these places neither lie on the earthquake occurring regions, not the people are affected, but are still tweeting the information to help the affected people. This may be due to their disaster awareness. Likewise, tweet locations were identified even out of the country. It shows that physical presence is not always required to help, inform, aware people; boundary may not makes the sense in the Cyber Geography. Most people were using English language to disseminate their feelings/messages, may be due to global understanding of the language as it might ease the rescue team to understand the situation.

Many Hashtags used were not standardized and were created by the individuals on their own. Such hashtags might be doubtful and might not convey right information to the concerned authority at the proper time thereby hampering the rescue operations. Standard hashtags like rapidresponseteam, NepalQuakeRelief and few others were initiated by the people/organizations working/supporting the rescue and recovery activities. Many linked urls with the tweets to give detail information about the earthquake may be because of 140character limit of the twitter.
Analysis shows that most of the tweets were tweeted when the earthquake of high magnitude occurred. This might represent that most of the people might be aware of the tremor and were spreading the information about it so that rescue operations could be done on right time.

Number of followers, friends, retweets and favorites are important indicators of information dissemination; having higher number of friends and followers may not always show that the status has been read by all. Having fewer friends, more followers and most of the status retweeted can be more communicative than having more followers, friends but no status retweeted or favorite. Also, Friends and followers might just read the status and not retweet it. There is a need to research further on this issue.

\section{CONCLUSION}

Twitter acted as an important communication medium during Nepal Earthquake 2015. Not all the tweets and hashtags convey right information; building standard messaging systems like creating standard hashtags for different disaster to reduce irrelevant and incorrect information and creating awareness among the twitter users help delivering right information to the concerned authority.

Nepal encountered various disasters like landslides and floods every year. Timely rescue has always been a challenging task because of lack of information on time; building standard messaging systems, spatial temporal analysis of the retrieved information and proper geovisualization can expedite rescue operations by providing on-time-information to the concerned authority to take prompt action thereby saving lives, properties and reducing the damages. Social media, with not much cost associated with it except for the mobile devices and internet, can be the quick, economical and effective means of communication during disasters if used properly.

\section{LIMITATIONS}

The data considered for the research includes data from 25 April 2015 to 18 May, 2015 and not all the data are included.

\section{ACKNOWLEDGEMENTS}

The author is thankful to Mr. Ming-Hsiang Tsou (Director of HDMA Center, Professor of Geography) and Jessica Dozier, Graduating Student at San Diego State University for providing Twitter data and helping with resources on Location Based Social Media.

\section{REFERENCES}

Cao and Wang et al., 2014. A Scalable Framework for Spatiotemporal Analysis of Location-based Social Media Data, Preprint submitted to Computers, Environment and Urban Systems September 10, 2014.

Chaffey, D., Global social media research summary 2016, 2016. Available from http://www.smartinsights.com/social-mediamarketing/social-media-strategy/new-global-social-mediaresearch/. 
Facebook, 2016. Stats 2015 [cited 15 March 2016]. Available from http://newsroom.fb.com/company-info/.

Goodchild, M.F., 2007. Citizens as sensors: the world of volunteered geography. GeoJournal 69 (4), 211-221.

HDMA, 2016. Center of Human Dynamics in the Mobile Age. 2016. HDMA "GeoViewer" and "SMART Dashboard" for Nepal Earthquake Disaster Response and Assistance San Diego State University 2015 [cited 15 March 2016]. Available from http://humandynamics.sdsu.edu/NepalEarthquake.html.

http://www.diva-gis.org/Data [cited 9 April, 2016].

${ }^{1} \mathrm{http}: / /$ www.seismonepal.gov.np/.

Internet World Stats, 2016. Asia Marketing Research, Internet Usage, Population Statistics and Facebook Information 2015 [cited 15 March 2016]. Available from http://www.internetworldstats.com/asia.html.

OCHA, 2014. 'Hashtag standards for emergencies', United Nations Office for the Coordination of Humanitarian Affairs, pp. $1-18$.

StatsMonkey, 2016. Mobile Facebook, Twitter, Social Media Usage Statistics in World 2015 [cited 15 March 2016]. Available from https://www.statsmonkey.com/table/21272-world-mobilesocial-media-usage-statistics-2015.php.

Tapia, A., Moore, K., \& Johnson, N., 2013. 'Beyond the trustworthy tweet: A deeper understanding of microblogged data use by disaster response and humanitarian relief organizations', pp. 770-779.

Thapa L., 2016. Social Media - Effective Means of Communication for Disaster Rescue and Recovery Activities, Newsletteer of Nepal Remote Sensing and Photogrammetric Society, Vol. VIII/April 2016.

Tsou et al., 2013a. 'Mapping ideas from cyberspace to real space: Visualizing the spatial context.

Tsou, M. H. and Leitner, M., 2013. Editorial: Visualization of Social Media: Seeing a Mirage or a Message? In Special Content Issue: Mapping Cyberspace and Social Media. Cartography and Geographic Information Science 40,2,55-60.

Tsou, M. H., 2015. Research challenges and opportunities in mapping social media and Big Data, Cartography and Geographic Information Science,

42:sup1, 70-74, DOI: 10.1080/15230406.2015.1059251.

Tsou, M. H. and Leitner, M., 2013. Editorial: Visualization of Social Media: Seeing a Mirage or a Message? In Special Content Issue: Mapping Cyberspace and Social Media. Cartography and Geographic Information Science 40,2,55-60.

Wikipedia, 2016. Facebook Safety Check. Wikipedia 2016 [cited 15 March 2016].

Zuckerberg, M., 2015. Mark Zuckerberg's Facebook Post 2015 [cited 15 March 2016]. Available from https://www.facebook.com/photo.php?fbid=101020608842382 $61 \&$ set=a.612287952871.2204760.4\&type=3\&theater. 